\title{
ORIGINAL
}

\section{EVALUACIÓN MÉDICA Y PSICOSOCIAL DE UNA POBLACIÓN ADULTA CON DISCAPACIDAD INTELECTUAL *}

\author{
José Almenara Barrios (1), Rodrigo García González-Gordon (2), José Pedro Novalbos Ruiz (1), \\ Beatriz Merello Martel (2), María José Abellan Hervás (3) y Cesáreo García Ortega $(1,4)$. \\ (1) Área de Medicina Preventiva y Salud Pủblica de la Universidad de Cádiz. \\ (2) AFANAS de Jerez de la Frontera. Cádiz. \\ (3) Inspección Médica Provincial. Instituto Social de la Marina de Cádiz. \\ (4) Gestoría de Usuarios del Hospital del S.A.S. de Algeciras. Cádiz. \\ $\left(^{*}\right)$ Este trabajo se ha realizado bajo un convenio Universidad Empresa, entre el Área de Medicina Preventiva de \\ la UCA y la asociación para atención del discapacitado AFANAS de Jerez de la Frontera
}

\section{RESUMEN}

Fundamento: El objetivo del presente trabajo es describir las características psicológicas, psicopatológicas, médicas y sociales de una población con discapacidad intelectual asistida en AFANAS-Jerez, que nos permita evaluar con una perspectiva epidemiológica este tipo de problema.

Método: Se realiza un estudio descriptivo de series de casos para caracterizar una población con retraso mental (RM). Se han evaluado las áreas psicológica, médica y social. El nivel intelectual se estudio mediante la Escala de Inteligencia de Weschler para adultos. La conducta adaptativa, la frecuencia, tipología y gravedad de los problemas de conducta se evaluaron mediante el Inventario para la Planificación de Servicios y Programación Individual. Se realizó una exploración psicopatológica de cribaje. El estudio se completa con una evaluación médica y social mediante entrevistas estructuradas. La información procedente de las distintas áreas se codifico y se anali$z o$ en los programas EPIINFO v6.0 y C.I.A..

Resultados: La edad media de la población con discapacidad intelectual estudiada es de 32,2 años (D.E. 9,2 años). Hay un $73 \%$ de varones frente a un $27 \%$ de mujeres. El $45,1 \%$ de los sujetos presentan problemas de comportamiento. Hemos establecido una relación estadística entre la ausencia o no de dichos problemas y el nivel de gravedad del RM, de forma que a mayor deterioro intelectivo mayor probabilidad de padecer los problemas de comportamiento. Dentro de los síntomas psicopatológicos destaca la presencia de ansiedad en un $60,2 \%$. La evaluación médica pone de manifiesto la gran cantidad de problemas asociados al RM. y así vemos como un $22,48 \%$ de los sujetos presentan epilepsia, casi el $20 \%$ Síndrome de Down. etc. Destacar también el bajo nivel educativo de los padres y los conflictos familiares que tienen su base en el RM del hijo.

Conclusiones: Se debe de señalar como el RM es una entidad muy compleja con una gran cantidad de trastomos médicos, psicológicos, psiquiátricos y sociales asociados, situación puesta de manifiesto en el presente estudio, estando muchos de los trastornos evidenciados relacionados con el nivel de gravedad del RM. Creemos que un abordaje epidemiológico en el estudio del RM puede ser útil para un mejor entendimiento del problema.

Palabras clave: Retraso mental. Epidemiologia. Comorbilidad. Minusvalías.

Correspondencia:

José Almenara Barrios C/ Estrecho, 15

11510 Puerto Real Cádiz

Correo electrónico: jose.almenara@uca.es
ABSTRACT

\section{Psychosocial-Medical Assessment of a Mentally Retarded Adult Population *}

Background: This study is aimed at describing the psychological, psychopathological, medical and social aspects of a mentally retarded population being provided with care by AFANAS-Jerez affording the possibility of assessing this type of problem from an epidemiological standpoint.

Methods: A descriptive case series study is conducted to characterize a mentally retarded (MR) population. An assessment was made of the psychological, medical and social aspects. The degree of intelligence was analyzed using the Weschler Adult Intelligence Scale. The adaptive behavior, frequency, typology and seriousness of the behavior problems were assessed by means of the Service Planning and Individual Scheduling Inventory. A psychopathological screening examination was conducted. This study is rounded out with a social and medical evaluation by means of structured interviews. The information from the different areas was encoded and was analyzed using the EPIINFO v6.0 and C.I.A. programs.

Results: The average age of the mentally retarded population studied was 32.2 (S.D. 9.2 years). Seventy-three percent $(73 \%)$ males as compared to $27 \%$ females. Behavior problems were found to exist among $45.1 \%$ of the subjects. We have set up a statistical relationship between the existence/non-existence of said problems and the degree of MR, such that the greater the loss of intellectual capacity, the greater the probability of having behavior problems. One of the psychopathological symptoms worthy of special mention is the anxiety found to exist among $60.2 \%$. The medical examination reveals the large number of problems related to with $\mathrm{MR}$, we thus finding $22.48 \%$ of the subjects to have epilepsy, nearly $20 \%$ Down's Syndrome, etc. Also worthy of special mention is the low educational level of the parents and the family conflicts resulting from the child's retardation.

Conclusions: Special mention must be made of how MR is a highly complex whole entailing a large number of related medical, psychological, psychiatric and social disorders, this being a situation brought to fore in this study, many of the disorders revealed to exist being related to the degree of MR. We are of the opinion that an epidemiological approach to the study of MR may be of use for a better comprehension of this problem.

Key words: Mental retardation. Epidemiology. Comorbility. Handicaps. 


\section{INTRODUCCIÓN}

Son pocos los estudios que tratan de evaluar con una perspectiva epidemiológica los problemas mentales y menos aún los que se centran en estudiar poblaciones con discapacidad intelectual o retraso mental (RM).

El RM es un término que se utiliza para caracterizar el bajo nivel de funcionamiento, tanto intelectual como adaptativo, de un individuo. La Asociación Americana para el Retraso Mental (AAMR) ${ }^{1}$ define al RM del siguiente modo. El RM se refiere a limitaciones sustanciales del funcionamiento actual. Se caracteriza por un funcionamiento intelectual significativamente por debajo del promedio, el cual se presenta junto a limitaciones asociadas en dos o más de las siguientes áreas de habilidades adaptativas: comunicación, autocuidado, vida en el hogar, habilidades sociales, uso de recursos comunitarios, autodirección, salud y seguridad, habilidades académicas funcionales, ocio y trabajo. El RM se presenta antes de los 18 años. Por su parte, la Asociación Americana de Psiquiatría (APA) en el manual diagnóstico y estadístico de los trastornos mentales (DSM-IV) ${ }^{2}$ incluye el RM dentro de los Trastornos del Desarrollo. Como sintomatología esencial de este grupo de trastornos destaca la alteración en el aprendizaje de las habilidades cognitivas, verbales, motoras o sociales.

Destacar, por último, que el RM es definido en la Clasificación Internacional de las Enfermedades (CIE) ${ }^{3}$, como trastorno definido por la presencia de un desarrollo mental incompleto o detenido, caracterizado principalmente por el deterioro de las funciones concretas de cada época del desarrollo y que contribuyen al nivel global de la inteligencia, tales como las funciones cognoscitivas, las de lenguaje, las motrices y la socialización.

Los síntomas esenciales del RM los podemos resumir en: Una capacidad intelectual general muy por debajo del promedio, que se acompaña de un déficit o deterioro significativo de la capacidad adaptativa, y con un comienzo antes de los 18 años. El diagnóstico se hace prescindiendo de si hay o no trastornos orgánicos $u$ otros trastornos mentales.

La clasificación del RM según niveles de gravedad, suele hacerse en función de la puntuación obtenida en una prueba de inteligencia. Según el cociente de inteligencia (CI), el DSM-IV (APA) ${ }^{2}$ clasifica al RM en leve (CI de 50-55 a 70), moderado (CI de 35-50 a 50-55), grave (CI de 20-25 a 35-40) profundo (CI por debajo de 20-25).

El RM leve corresponde a la categoría pedagógica de «educable». Este grupo reúne a una amplia capa de población afectada por este trastorno, alrededor del $85 \%$. En la actualidad toda persona con RM leve puede vivir perfectamente adaptada a su comunidad, vivir de forma independiente o en apartamentos supervisados o en viviendas agrupadas, a menos que exista algún trastorno asociado que impida estas posibilidades.

El RM moderado constituye el $10 \%$ de las personas afectadas. Pueden también beneficiarse del aprendizaje de habilidades sociales y laborales, aunque los que siguen estudios son incapaces por lo general de progresar más allá del nivel de segundo grado. En la vida adulta, pueden contribuir a su propio mantenimiento, efectuando trabajos que no requicran una gran habilidad bajo estrecha supervisión en talleres protegidos o en el mercado de trabajo. Necesitan orientación y supervisión cuando se encuentran en situaciones de estrés. Se adaptan bien a la vida comunitaria.

El grupo con RM grave constituye el $3-4 \%$ de la población con RM. En la vida adulta, pucden hacer tarcas scncillas bajo una estrecha supervisión. Muchos se adaptan perfectamente a la vida de la comunidad, en viviendas protegidas o con sus familias, a menos que tengan una dificultad asociada 
que requiera atención especializada u otro tipo de cuidados.

El grupo con RM profundo está formado aproximadamente por el 1-2\% de la población con RM. Durante los primeros años estos niños muestran una capacidad mínima para el funcionamiento sensorial y motriz. Requieren para un óptimo desarrollo, un entorno altamente estruclurado, con ayuda y supervisión constantes. El desarrollo motor, la autonomía y las habilidades de comunicación pueden perfeccionarse con un entrenamiento adecuado.

Los principales factores etiológicos se pueden agrupar en: factores hereditarios, alteraciones tempranas del desarrollo embrionario, problemas durante la gestación o prenatales, trastornos somáticos que se presentan durante la niñez e influencia del entorno o de otros trastornos mentales.

En cuanto a la prevalencia del RM los estudios más recientes la sitúan en $1 \%$, siendo más frecuente entre los niños que entre las niñas $(1,5: 1)^{4}$. También se observa que la prevalencia de otros trastornos es mayor entre niños con RM que en la población general.

La evaluación psicológica del RM se sustenta en tres pilares básicos: La evaluación de la inteligencia, la evaluación de la capacidad adaptativa y la exploración psicopatológica de cribaje ${ }^{5}$. Según los criterios de la APA la capacidad Intelectual General se define como Cociente Intelectual (CI, o equivalentes a $\mathrm{CI}$ ), obtenidos mediante la evaluación de una o más personas a través de un test de inteligencia administrado individualmentc. Uno de los instrumentos más ampliamente reconocidos para la evaluación de la inteligencia es la Escala de Inteligencia de Weschler para Adultos (WAIS). Cuando esta no se puede administrar bien por niveles muy bajos de $\mathrm{Cl}$, dificultades graves de comunicación o deficiencia sensorial, se estima en función de la gravedad de los déficits en las áreas de conducta adaptativa ade- más del juicio clínico. La capacidad adaptativa es la eficacia que muestra la persona en determinadas áreas de su comportamiento, como habilidades sociales, comunicación y habilidades para resolver problemas cotidianos, y a lo adecuado de sus respuestas en relación con la independencia personal y a la responsabilidad social esperados por su edad y grupo cultural. La capacidad adaptativa de las personas con RM (y las que no lo tienen) se ve influenciada por las características de personalidad, el grado de motivación, el nivel de educación, y por las oportunidades vocacionales y sociales. La conducta adaptativa puede mejorarse con los esfucrzos adecuados, mientras el CI tiende a permanecer más estable.

La conducta adaptativa y la inteligencia son dos conceptos que están relacionados pero no deben confundirse. La conducta adaptativa debe ser concebida en términos de destrezas necesarias para ser miembros funcionalmente activos de una sociedad. Se encuadraría, dentro de la «intcligencia práctica», es decir, la capacidad para valerse por uno mismo de forma independiente, en actividades de la vida diaria (comida, vestido, higiene, evitación de peligros...). El origen del interés actual por medir la conducta adaptativa surge como reacción contra el $\mathrm{Cl}$ entendido como equivalente a una medida global de conducta humana. Se pretende proporcionar una perspectiva diferente a la de los tests de inteligencia clásicos, evitando sesgos y el efecto nocivo de las etiquetas. La conducta adaptativa y la tipología, frecuencia y gravedad de los problemas de conducta se evalúan mediante la administración del ICAP (Inventario para la Planificación de Servicios y Programación Individual) ${ }^{6}$.

En cuanto a la exploración psicopatológica de cribaje cs necesaria en tanto que la mayoría de las revisiones coinciden en que los trastornos psiquiátricos se observan más frecuentemente en este grupo de enfermos que en la población general. La mayoría de las estimaciones de la prevalencia de tras- 
tornos psiquiátricos graves, incluyendo tanto los trastornos de personalidad como la psicosis, están entre el 8 y el $15 \%$. Cuando se incluyen trastornos emocionales menores, las estimaciones se disparan en torno al $50 \%{ }^{7}$. La exploración psicopatológica de cribaje se apoya en varias secciones de la Entrevista de Evaluación Psiquiátrica de Adultos con Trastornos del Desarrollo (PAS-ADD) ${ }^{8}$, que tuvo como propósito inicial el cribaje de poblaciones vulnerables a padecer trastornos psiquiátricos.

Es necesaria la evaluación médica en sujetos con deficiencia intelectual, ya que frecuentemente el origen de esta discapacidad es una enfermedad genética o pediátrica que condiciona la aparición de un síndrome clínico complejo. La aparición de esta clínica no es, generalmente, aguda sino que va a depender en gran medida de la edad del sujeto. Esto justifica plenamente la evaluación médica continuada con fines no ya curativos, sino preventivos que detecten de forma precoz los problemas de salud más prevalentes.

El presente estudio se enmarca en una línea de trabajo conjunta entre AFANAS Jerez. [Asociación de Asistencia a Minusválidos Psíquicos de Jerez de la Frontera (Cádiz)], organización no gubernamental sin ánimo de lucro, de ámbito de actuación local, con más de treinta años de experiencia en la asistencia a personas con discapacidad intelectual y miembro de la Confederación Española de Asociaciones Pro Minusválidos Psíquicos (FEAPS) y el Área de Medicina Preventiva y Salud Pública de la Universidad de Cádiz, dentro de un convenio Universidad-Empresa. Con un primer objetivo que es el que nos hemos trazado para la realización del trabajo, describir las características sociodemográficas, psicológicas, psicopatológicas y médicas de una población con discapacidad intelectual (RM) asistida en AFANAS-Jerez, que nos permita evaluar con una perspectiva epidemiológica este tipo de problemática.

\section{MATERIAL Y MÉTODO}

Se presenta un estudio descriptivo de series de casos para caracterizar epidemiológicamente una población asistida con RM. Se evalúan las siguientes áreas: psicológica, médica y social.

Dentro de la evaluación psicológica se estudio en primer lugar la evaluación de la inteligencia a través de la Escala de Inteligencia de Weschler para adultos (WAIS).

Esta prueba no se pudo realizar a un porcentaje de la población por presentar niveles muy bajos de capacidad intelectual, o con graves dificultades de comunicación. En estos casos, el nivel de RM se estimó en función de la gravedad de los déficits en las áreas de conducta adaptativa además del juicio clínico correspondiente.

La conducta adaptativa y la tipología, frecuencia y gravedad de los problemas de conductas se evaluaron mediante la administración del ICAP. La recogida de datos se informatiza en el Programa Informático para la Corrección y Base de Datos del ICAP (Compuscore-ICAP) ${ }^{6}$. Para cumplimentar los cuestionarios se utilizó la información proporcionada tanto por los familiares como por el personal de atención directa a los sujetos.

La exploración psicopatológica de cribaje se apoyó en el Cuestionario Previo a la administración de la Entrevista de Evaluación Psiquiátrica de Adultos con Trastornos del Desarrollo (PAS-ADD 10) ${ }^{8}$.

El estudio se completo con una evaluación médica, basada en una entrevista clínica estructurada y una exploración física con revisión de pruebas complementarias. La entrevista clínica estructurada constó de los siguientes puntos: antecedentes familiares y personales (con inclusión específica de patología prenatal), hábitos tóxicos, ingresos $\mathrm{c}$ intervenciones quirúrgicas previas, estado clínico y medicación actual. Se realizo una exploración física por aparatos 
y sistemas con inclusión de exploración bucal. Y se contrastó y evaluó los informes médicos anteriores que presentaban los familiares o que obraban en posesión de la institución, fundamentalmente la calificación de minusvalia remitida por los Equipos de Valoración y Orientación (E.V.O.) emitida para el $100 \%$ de los sujetos estudiados. Como consecuencia de esta evaluación médica se obtiene de forma básica para cada usuario un diagnóstico médico principal y hasta cuatro patologías asociadas. De los informes evaluados se obtuvieron las cifras correspondientes a los sujetos que nacieron con bajo peso, por debajo de 2500 gr, prematuridad, menos de 37 semanas de gestación, o los diagnósticos de hipoxia o encefalopatia hipoxica. Los sujetos que se detectaron con posibles problemas motores, fueron sometidos a evaluación fisioterapeutica.

Se realizó también una evaluación social, mediante visita domiciliaria por parte de una trabajadora social recogiendo datos de cobertura asistencial, económicos, familiares, entorno e historia social, así como aspectos prácticos de vivienda, nivel de compromiso con la institución o su grado de satisfacción con la misma.

Toda la información recogida se volcó en una base de datos confeccionada en el programa EPIINFO v $6.0^{\circ}$. El cuestionario creado consta de 217 variables codificadas, clasificadas en tres grandes grupos: sociodemográficas, médicas y del historial psicopatológico. Se realizaron los análisis estadísticos pertinentes: medidas de síntesis habituales de centralización y dispersión y se calcularon los correspondientes intervalos de confianza al $95 \%\left(\mathrm{IC}_{95 \%}\right)$ cuando fueron necesarios mediante el programa informático CIA ${ }^{10}$. Como test de hipótesis se utiliza la prueba $\chi^{2}$ o el test exacto de Fisher en las situaciones necesarias y como medida de riesgo la odds ratio (OR). Siempre se trabajo con un nivel de confianza del $95 \%$.

\section{RESULTADOS}

Presentamos una primera valoración de los resultados obtenidos tras estudiar los 129 sujetos con discapacidad intelectual asistidos en AFANAS-Jerez. Dicha población es relativamente joven, con una edad media de 32,2 años (DE 9,2 años). Encontramos un 73\% de varones frente a un $27 \%$ de mujeres, con una relación varón mujer de $3: 1$. La edad media de las mujeres es discretamente inferior a la de los varones, 31,5 años (DE 7,9 años) frente a 32,5 años (DE 9,6 años).

Se estudio en primer lugar la distribución de la población según niveles de discapacidad intelectual, presentado los resultados que aparecen en la tabla 1,se observa una discordancia entre los valores según niveles de gravedad entre la población general y los observados en AFANAS. La población asistida en AFANAS cuenta con un número muy superior de sujetos gravemente afectados, en comparación con la prevalencia estimada en la población general y un menor número de sujetos en el nivel leve.

Tabla 1

Distribución de la población con RM según niveles de gravedad. Comparación con una población general con RM

\begin{tabular}{|lcc|}
\hline Grado de RM & $\begin{array}{c}\text { Población General } \\
\text { con RM. (DSM-IV) } \\
(\%)\end{array}$ & $\begin{array}{c}\text { Población } \\
\text { estudiada } \\
(\%)\end{array}$ \\
\hline Leve & 85 & 32 \\
Moderado & 10 & 39,6 \\
Grave & $3-4$ & 20,7 \\
Profundo & $1-2$ & 7,5 \\
\hline
\end{tabular}

Los problemas de comportamiento pudieron estudiarse en 122 sujetos del total, presentando tales problemas 55, es decir el $45,1 \%\left(\mathrm{IC}_{95 \%}\right.$ 36,3-53,9). La tipología de los problemas del comportamiento se puede ver en la tabla 2. Hemos observado también como los mencionados problemas de comportamiento se incrementan con la edad de los individuos. 
Tabla 2

Tipología de los problemas de comportamiento

\begin{tabular}{|lccc|}
\hline $\begin{array}{l}\text { Problema de } \\
\text { comportamiento }\end{array}$ & Frecuencia & $\%$ & IC 95\% \\
\hline Autulesión & 23 & 18,9 & $11,9-25,8$ \\
Conductas destructivas & 24 & 19,7 & $12,6-26,7$ \\
Heteroagresividad & 35 & 28,7 & $20,7-36,7$ \\
Comportamiento social & & & \\
ofensivo & 48 & 39,3 & $30,7-48$ \\
Estereotipias & 69 & 56,6 & $47,8-65,4$ \\
Retraimiento & 70 & 57,4 & $48,6-66,2$ \\
Conductas disruptivas & 70 & 60,7 & $52-69,3$ \\
\hline
\end{tabular}

IC: Intervalo de confianza.

Un análisis que nos ha parecido oportuno es el encaminado a establecer una relación entre el nivel de gravedad del RM y los problemas de comportamiento explicitados. Se constata una relación estadística entre la ausencia o no de problemas de conducta en global y el nivel de gravedad del RM, de forma que a mayor deterioro intelectivo existe una mayor probabilidad de padecer los problemas referidos $\left(\chi^{2}=20,49\right.$, g.l. $=4$, $\mathrm{p}=0,000)$. Confirmándose cuando agrupamos las clases de RM en dos, leves y moderadas por un lado y graves y profundas por otro, obteniéndose una $\mathrm{OR}$ de 7,23 $\left(\mathrm{IC}_{95 \%} 2,67-19,6\right)$. Las asociaciones entre los distintos problemas de comportamiento y el RM se pueden observar en la tabla 3.

Tabla 3

Asociación entre nivel RM y los problemas de comportamiento

\begin{tabular}{|lccc|}
\hline $\begin{array}{l}\text { Problema de } \\
\text { comportamiento }\end{array}$ & Significación & OR & IC 95\% \\
\hline Heteroagresividad & 0,04 & 2,40 & $0,92-6,30$ \\
Destrucción de objetos & 0,02 & 3,30 & $1,16-9,45$ \\
Autolesión & 0,002 & 4,61 & $1,59-13,55$ \\
Social Ofensivo & 0,01 & 3 & $1,18-7,73$ \\
\hline
\end{tabular}

OR: odds ratio.

IC $95 \%$ : Intervalo de confianza de la odds ratio.

Hemos intentado también determinar la prevalencia de los distintos síntomas psicopatológicos, pese a las dificultades inheren- tes a no existir un instrumento estandarizado adaptado al español para la exploración psiquiátrica de sujetos adultos con RM. El síntoma psicopatológico mas observado ha sido la ansiedad presente en un $60,2 \%$ de los sujetos. La distribución de la población según la presencia de síntomas psicopatológicos se presenta en la tabla 4 . No hemos encontrado asociación estadística entre cl nivel de RM y la ansiedad o los síntomas psicóticos, pero esta se manifestaba fundamentalmente con los síntomas autistas (Prueba exacta de Fisher: $p=0,00001, O R=13,75$ ) y en menor medida con los trastornos del estado de ani$\operatorname{mo}\left(\chi^{2}=9,53\right.$, g.l. $\left.=4, p=0,049, O R=3,16\right)$.

Tabla 4

Distribución de los síntomas psicopatológicos

\begin{tabular}{|lll|}
\hline Síntomas psicopatológicos & $\%$ & IC $95 \%$ \\
\hline Ansiedad & 60,2 & $51,0-71,2$ \\
Trastornos del estado de animo & 23,7 & $16,2-34,4$ \\
Autismo & 13,9 & $7,15-19,9$ \\
Sintomas psicóticos & 12,6 & $6,95-19,4$ \\
\hline
\end{tabular}

IC: Intervalo de Confianza.

La evaluación médica, constata las plurideficiencias, que acontecen en este colectivo. Dentro de los antecedentes personales destacamos el número importante de sujetos con hermanos afectados de oligofrenia, $18,4 \%\left(\mathrm{IC}_{95 \%} 11,3-27,5\right)$, que puede orientar a la existencia de una base genética de un importante número de casos. Del resto de antecedentes familiares, sobresale el alcoholismo en los padres presente en un $25 \%$ ( IC $\left._{95 \%} 15,8-36,3\right)$ y los antecedentes de trastornos afectivos 7,2\% ( $\left.\mathrm{IC}_{95 \%} 2,95-14,3\right)$ o neurosis $6,2 \%\left(\mathrm{IC}_{95 \%}, 2,33-13,1\right)$ en las madres.

Al estudiar los factores etiológicos del retraso mental destaca fundamentalmente la existencia de alteraciones cromosómicas, presentes en un $26,3 \%\left(\mathrm{IC}_{95 \%} 17,8-36,3\right)$ de los sujetos, siendo el Sdr. de Down con 25 sujetos $\left(19,35 \%\right.$ del total.IC $\left.\mathrm{C}_{95 \%} 12,6-26,2\right)$ la 
entidad más característica. Dentro de lo que podemos catalogar como factores de riesgo o trastornos adquiridos que pueden explicar el desarrollo del retraso mental, hemos encontrados prevalencias elevadas de bajo peso al nacer $14 \%\left(\mathrm{IC}_{95 \%} 7,87-22,4\right)$, hipoxia o encefalopatia anóxica $35,3 \%\left(\mathrm{IC}_{95 \%} 26-44,6\right)$ y prematuridad con un $8,2 \%\left(\mathrm{IC}_{95 \%} 3,59-15,4\right)$. Evidenciando la posibilidad de sufrimiento fctal en cl parto con el consiguiente riesgo de lesiones hipoxico-isquemicas. La patología postnatal no aporto resultados relevantes aunque luego en la discusión haremos un comentario al respecto.

Se estudiaron también los hábitos tóxicos de nuestra población no encontrando diferencias llamativas con los de la población general, la proporción de sujetos que se declaran fumadores es de un $21,8 \%\left(\mathrm{IC}_{95} \%\right.$ $14,1-29,5)$, con consumo medios no muy elevados ( 9,5 cigarrillos/día) aunque una cuarta parte fuma mas de 20 cigarrillos/día. El tiempo medio de consumo es de 13,6 años. Respecto al consumo de alcohol, se reconocen consumidores habituales el $11 \%$ $\left(\mathrm{IC}_{95 \%} 5,13-16,9\right)$ de los sujetos, no encontrando asociación significativa con los antecedentes de alcoholismo en los padres. Aunque hablemos de consumo habitual no podemos hablar de consumos elevados en esta población.

Un dato relevante también es la elevada frecuencia de problemas alérgicos que llega al $39,5 \%$, o el número de intervenciones quirúrgicas presentes en la mitad de los sujetos.

Los hallazgos en la exploración física y fisioterapeutica señalan la pluridad de problemas presentes, que se recogen en la tabla 5. Por otro lado se constato también un $6 \%$ de sujetos hipertensos, 4 sujetos con bronquitis asmática, 4 diabéticos y casos aislados de hipotiroidismo y de parálisis facial.

En relación a las condiciones o handicaps asociados destacamos la presencia de 29 sujetos con epilepsia $\left(22,48 \% \mathrm{IC}_{95 \%}\right.$
Tabla 5

Distribución de problemas físicos encontrados en la exploración

\begin{tabular}{|lrrc|}
\hline $\begin{array}{l}\text { Hallazgos } \\
\text { exploratorios }\end{array}$ & Frecuencia & $\%$ & IC 95\% \\
\hline Alteraciones del tono mus- & & & \\
cular & 29 & 22,48 & $15,3-29,7$ \\
Boca Séptica & 24 & 18,6 & $11,9-25,3$ \\
Alteraciones de la Agude- & & & \\
za visual & 22 & 17,05 & $10,6-23,5$ \\
Disquinesias & 14 & 10,85 & $5,49-16,2$ \\
Alteraciones del equilibrio & 14 & 10,85 & \\
Pies planos & 10 & 7,75 & $3,78-13,8$ \\
Cuello Corto & 5 & 3,87 & $1,28-8,81$ \\
Hipoacusia & 3 & 2,32 & $0,48-6,65$ \\
Dismetria & 3 & 2,32 & $0,48-6.65$ \\
Adenopatias bilaterales del & & & \\
cuello & 2 & 1,55 & $0,18-5,49$ \\
Insuficiencia venosa & 2 & 1,55 & $0,18-5,49$ \\
Paladar hendido & 1 & 0,77 & $0,018-4,24$ \\
Hernia abdominal & 1 & 0,77 & $0,018-4,24$ \\
IIernia inguinal & 1 & 0,77 & $0,018-4,24$ \\
Pies cavos & 1 & 0,77 & $0,018-4,24$ \\
Piernas en X & 1 & 0,77 & $0,018-4,24$ \\
Sindactilia & 1 & 0,77 & $0,018-4,24$ \\
Genu valgo & 1 & 0,77 & $0,018-4,24$ \\
\hline
\end{tabular}

IC: Intervalo de confianza.

$15,3-29,7)$ y un $3 \%$ con parálisis cerebral $\left(\mathrm{IC}_{95 \%} 0,50-7,01\right)$. También un $10,8 \%$ de los sujetos presentan manifestaciones de problemas neurológicos como hemiparesia, paraparesia, hemiplejía o paraplejía.

Hemos detectado también que el 17,05\% de los sujetos presentan alteraciones de la agudeza visual y un $2,32 \%$ presentaron hipoacusia.

Dentro de la evaluación social realizada destacamos dos resultados que mediatizan en gran medida la asistencia y que se deben tener en cuenta, por un lado el bajo nivel educativo de los de los padres, no completando los estudios primarios el $54,9 \%\left(\mathrm{IC}_{95 \%} 41,8-63,9\right)$ de los padres y el $71 \%\left(\mathrm{IC}_{95 \%} 62,8-79,8\right)$ de las madres. Por otro lado hemos detectado que en el 20,2\% ( IC $_{95 \%}$ 12,7-27,3) de las familias se instauran conflictos derivados de la discapacidad intelectual del hijo. 


\section{DISCUSIÓN}

Existe una discordancia entre la distribución de nuestra población con RM según niveles de gravedad y la que se da en la DSM-IV, tabla 1. Se observa que en la población general con RM existe un porcentaje muy superior de sujetos con RM leve e inferior de RM grave o profundo. Esto puede ser debido a una menor atención asistencial a los individuos con RM leve, bien por que pasen desapercibidos en ambientes socialmente deprimidos o bien por un intento de ocultación del problema que no favorece su asistencia en organizaciones como la estudiada o bien porque utilizan vías alternativas de integración. Por el contrario, nos encontramos con porcentajes muy superiores a los de la población general con RM, en los grados grave y profundo, más difíciles de ocultar y con necesidades imperiosas de asistencia a todos los niveles.

Al determinar los problemas de comportamiento observamos como la distribución por tipologías es similar a la que aparece en la literatura. Sovner ${ }^{11}$ ha estudiado dichos problemas en poblaciones con RM, encontrando un porcentaje de enfermos que presentan algún problema relacionado con la agresión, que oscila entre el $11 \%$ y el $25 \%$, manifestando las cotas más altas los sujetos institucionalizados y los gravemente afectados, siendo compatibles nuestros resultados con los mencionados por este autor. El mismo autor nos presenta una prevalencia de comportamientos autolesivos que oscila entre el $2,6 \%$ en sujetos con deficiencias leves y el $16,9 \%$ en sujetos con minusvalias profundas e institucionalizados. Si bien nosotros presentamos una prevalencia discretamente superior $(18,9 \%)$, hemos de tener en cuenta que no trabajamos con población general de sujetos con RM, sino con una población asistida en una organización determinada y ya hemos comentado que difieren en cuanto a la distribución del nivel de gravedad de RM.

En nuestro estudio ponemos en evidencia la comorbilidad de los trastornos de com- portamiento que rara vez se dan solos, la mayoría de los autores han descrito también esta característica ${ }^{12}$.

En distintos estudios ${ }^{12,13}$ se pone de manifiesto la relación entre los mencionados problemas de comportamiento y el nivel de discapacidad intelectual. Nosotros con un análisis de riesgos habitual en epidemiología pero no existente en los trabajos consultados, hemos cuantificado dicha relación, como se observo en la tabla 3 mediante las OR y los correspondientes intervalos de confianza. El mayor riesgo se estimo en las situaciones de autolesiones, si bien el intervalo de confianza de la OR es amplio como consecuencia de la estratificación en el análisis. Le siguen ya con intervalos mas cerrados la destrucción de objetos y las situaciones denominadas social ofensiva.

El estudio de la sintomatología psicopatológica y la evaluación psiquiátrica con profundidad, que como ya hemos comentado en el capítulo de resultados es altamente complicada en la población adulta con discapacidad intelectual, conlleva una serie de dificultades metodológicas y prácticas derivadas de los déficits cognitivos y lingüísticos habitualmente presentes en esta población. E1 problema se dificulta al no existir ningún instrumento estandarizado adaptado al español para la exploración psiquiátrica de sujetos adultos con retraso mental. Como consecuencia de lo mencionado la literatura muestra variaciones a la hora de estimar la prevalencia de los distintos síntomas psicopatológicos. Campbell y Malone ${ }^{14}$ en una revisión sobre los estudios acerca de los índices de prevalencia de trastornos mentales en poblaciones con RM detectan una variación que va del $14,3 \%$ al $67,3 \%$. No solo las dificultades apuntadas son las causantes de las variaciones en las estimaciones, sino que también distintos criterios diagnósticos o de clasificación apuntan a explicar la gran variabilidad en los resultados, de tal forma que los estudios que incluyen los trastornos de conducta dentro de los síntomas psicopatólogicos desembocan en prevalencias elevadas ${ }^{15}$. Lo 
que parece estar claro es que las personas con RM tienen casi dos veces más probabilidades de desarrollar una enfermedad mental que la población normal ${ }^{16}$. En nuestra población de estudio como aparece en la tabla 4, son numerosos los síntomas psicopatológicos detectados, encontrando cifras similares a los estudios revisados incluyendo la sintomatología psicótica donde estimamos una cifra del 12,6\% ( IC $\left._{95 \%} 0,0695-0,194\right)$, dentro de la que estiman Fraser y Nolan ${ }^{7}$ para este grupo de pacientes.

La evaluación médica constata la base genética de muchos de los casos estudiados y la existencia de antecedentes de sufrimiento fetal en muchas ocasiones. Decíamos en los resultados que el estudio de la patología postnatal no aporto datos relevantes, sin embargo puede ser importante el sesgo de memoria o información, ya que los cuidadores de estos pacientes pueden considerar poco importante patologías médicas en el contexto de un deterioro intelectual, que se erige como el gran protagonista. Por otro lado la evaluación médica pone de manifiesto que las necesidades de cuidados médicos en esta población presenta especiales peculiaridades debido a las dificultades de diagnóstico y tratamiento ocasionadas en gran medida por las dificultades de comunicación que hace necesario la especialización de sus terapeutas. De todas formas, pensamos que la asistencia primaria puede y debe prestar una gama de actividades de prevención y promoción de la salud importantes, ya que representan grupos especiales de riesgo para patologías ya conocidas (cardiopatías, epilepsia, etcétera). Además, los problemas de salud más prevalentes de esta población con discapacidad intelectual son susceptibles de pruebas de screening muy eficientes; pruebas de agudeza visual, pruebas de Rinne y Weber para la hipoacusias, electrocardiograma y auscultación cardiaca en sujetos con Síndrome de Down, etcétera.

En el presente trabajo ponemos en evidencia que tanto los trastornos psicológicos como los médicos, recordemos las cifras ob- tenidas para trastornos como la epilepsia, las lesiones neurológicas, etcétera, asociados a la discapacidad intelectual son múltiples, y que junto con un entorno social desfavorable condicionan en gran medida la asistencia. Creemos que las evaluaciones aisladas (médicas, psicológicas, psiquiátricas, etcétera.) resultan en determinadas ocasiones de una elevada especificidad pero muy poco sensibles parta la detección o cribado completo de la problemática de nuestros usuarios. Como consecuencia, el conocimiento epidemiológico de la población con discapacidad intelectual permitirá establecer prioridades en la atención del RM, que pensamos debe tender a un modelo asistencial de integración y promoción de estas personas ${ }^{17}$, en el cual la epidemiología debe decir algo.

\section{AGRADECIMIENTOS}

Son muchas las personas que han colaborado en este trabajo, pero de una manera especial quisiéramos agradecer la colaboración prestada por María Isabel Baraza, David Gómez y Gema Vicenti en la informatización de los datos médicos. A la psicóloga Ana Solís por su inestimable ayuda en la evaluación psicológica y al fisioterapeuta Juan Palmero por su labor en la exploración y evaluación física.

\section{BIBLIOGRAFÍA}

1. American Association on Mental Retardation (AAMR). Mental Retardation: Definition, classification and systems of supports. $9^{\text {th }} \mathrm{ed}$. Washington: American Association on Mental Retardation; 1992.

2. American Psychiatric Association. Diagnosis and Statistical Manual of Mental Disorder. $4^{\text {th }}$. Edn. Reviewed (DSM-IV). Washington: American Association on Mental Retardation; 1993.

3. Clasificación Internacional de Enfermedades.9." Revisión, Modificación Clínica. Madrid: Ministerio de Sanidad y Consumo; 1994.

4. Van Schrojenstein Lantman-de Valk. Prevalence and incidence of health problems in people with 
intellectual disability. J. Intellectual Disability Research 1997;41:42-51.

5. Salvador L, García MJ, Romero C, Montero S. Evaluación psicosocial del retraso mental. Madrid: Ministerio de Asuntos Sociales-INSERSO; 1995.

6. Montero D. Evaluación de la conducta adaptativa en personas con discapacidades. Adaptación y validación del ICAP. Recursos e instrumentos psicopedagógicos. Bilbao: Departamento de investigación y evaluación educativa ICE. Deusto: Ediciones Mensajero S.A; 1993.

7. Fraser W, Nolan M. Psychiatric disorders in mental retardation. En: Bouras N, editor. Mental Health in Mental Retardation. Recent advances and practices. Cambridge: Cambridge University Press; 1994.

8. Moss SC, Godberg D, Patel P, Prosser H, Ibbotson B, Simpson N, Rowe S. The psychiatric assessment schedule for adults with developmental disability (PAS-ADD). Hester Adrian Research Centre and the institute of psychiatry; 1995.

9. Epilnfo v6.02 (programa de ordenador) Center for Disease Control \& Prevention. Geneva: World Health Organization; 1994.

10. Martin J. Gardncr. Confidence Interval Analysis v1.0 (programa de ordenador) \& BMJ;1989.
11. Sovner R, Hurley AD. Assessment tools which facilitate psychiatric. Evaluations and treatment. The Habilitative Mental Healthcare Newsletter 1990;9:11

12. Emerson E. Analysis and intervention in people with learning dificultase. Cambridge: Cambridge University Press; 1995.

13. Holt G. Challenging Behaviour. En: Bouras N, editor. Mental Health in Mental Retardation. Recent advances and practices. Cambridge: Cambridge University Press; 1994.

14. Campbell M, Malone RP. Mental retardation and psychiatric disorders. Hosp Community Psychiatry 1991;4:42.

15. Moss S. Methodological issues in the diagnosis of psychiatric disorders in adults with learning disability. Thornfield JI 1995;18:9-18.

16. Fletchen RJ. Mental health services for mentally retarded persons: A community mental health center model. En: Dosen editores. Treatment of mental illness and behavioural disorden in the mentally retarded. Amsterdam: Logon publications; 1990 .

17. Pérez J, Alonso F, Salvador L. Integración y promoción de las personas con retraso mental. Cabra (Córdoba). Córdoba: PROMI;1997. 